Schweiz. Gastroenterol. 2020 1 1:130-137 https://doi.org/10.1007/s43472-020-00026-8 Online publiziert: 23. November 2020 (c) Der/die Autor(en) 2020
Daniela Cabalzar-Wondberg · Matthias Turina

Klinik für Viszeral- und Transplantationschirurgie, Universitätsspital Zürich, Zürich, Schweiz

\title{
Mesenchymale Stammzellen als neue Therapie bei Morbus- Crohn-Patienten mit Fisteln
}

u. a. aufgrund der oft putriden, stinkenden Sekretion, Schmerzen und der rezidivierenden schmerzhaften Entzündungen [6].

Die Behandlung perianaler Fisteln bei Patienten mit einem Morbus Crohn unterscheidet sich aufgrund einiger krankheitsbedingter Faktoren deutlich von der Behandlung ohne zugrunde liegenden Morbus Crohn. Bei letzteren sind die Fisteln in der Regel kryptoglandulären Ursprungs, wohingegen Crohnassoziierte Fisteln aufgrund eines durch chronische Inflammation verursachten Epitheldefekts auftreten [7].

Bei Patienten mit einem perianalen Morbus Crohn liegen in bis zu 70-80\% der Fälle komplexe Fisteln vor $[4,8]$. Diese unterscheiden sich analog der ParksKlassifikation von den einfachen Fisteln durch die Anzahl ihrer Fistelöffnungen mit häufig mehreren Öffnungen, ihren Verlauf (hoher transsphinktärer Verlauf mit einer betroffenen Sphinktermuskulatur von über einem Drittel, supra- und extrasphinktäre Fisteln), durch das Auftreten rektovaginaler Fisteln, eine Analstenose oder gleichzeitige intraluminale Inflammation $[9,10]$.

Gut $71 \%$ der Patienten mit einem perianalen Morbus Crohn werden mindestens einmal reoperiert und ein Drittel der Patienten benötigt im Krankheitsverlauf einen anderweitigen grossen operativen Eingriff [1]. Durch die wiederholten Voroperationen, sei es nach Abszessexzision oder Fisteloperation, präsentieren sich die Patienten zudem oftmals mit einer ausgeprägten Narbenbildung und ggf. einer Analkanalstenose [10], was ein chirurgisches Vorgehen weiter erschwert.
Zusätzliche Herausforderungen für einen erfolgreichen chirurgischen Eingriff bestehen durch die luminale Inflammation und die begleitende medikamentöse Immunsuppression.

Auch bei optimaler medikamentöser und chirurgischer Therapie kommt es bei etwa $16 \%$ der Patienten zu einem Rezidiv der Fistel nach einem Jahr. Das Rezidivrisiko steigt im Verlauf der Krankheitsdauer weiter an mit einer Inzidenz von $31 \%$ nach 3 Jahren und $40 \%$ nach 5 Jahren $[11,12]$.

Die Erstmanifestation der perianalen Fistel(n) ist häufig ein Abszess, aber auch bereits vorhandene Fisteln können durch wiederholte Abszedierungen symptomatisch werden. Abszesse gehören in aller Regel chirurgisch eröffnet. Meist wird anschliessend antibiotisch behandelt, der langfristige Erfolg hinsichtlich der Fistelheilung bleibt jedoch häufig aus und der Benefit der Behandlung nimmt rapide ab nach Stoppen der antibiotischen Therapie [13].

Die aktuell gängigen und evidenzbasierten Operationstechniken für den Verschluss kryptoglandulärer Fisteln können ebenfalls bei Crohn-assoziierten Fisteln angewendet werden, sofern die lokalen Gegebenheiten den Einsatz dieser Techniken erlauben.

Bei Crohn-Fisteln liegt die Heilungsrate bei einem Verschluss mittels eines Advancement Mukosaflap bei ca. $64 \%$ [14]. Gemäss den Daten eines Systemic Review von Rozalén et al. kommt es bei einem Drittel der initial erfolgreich mittels Mukosaflap behandelten Patienten jedoch zu einem Rezidiv [15]. Eine Inkontinenz nach diesem operativen Verfahren wird mit 7,8\% beziffert [16]. 
Das Ligation-of-the-intersphinctericfistula-tract(LIFT)-Verfahren zeigt eine Heilungsrate in der Literatur um $67 \%$ [17]. Daten betreffend Rezidivraten bei Patienten mit Morbus Crohn gibt es in der Literatur jedoch nur wenige. Es finden sich kleine Fallserien wie z. B. die retrospektive Kohortenstudie von van Praag et al., die Rezidivrate wird hier mit 21,1\% angegeben [18].

Der Anal Fistula Plug sowie der „over the scope clip (OTSC) proctology" haben nach anfänglich vielversprechenden Resultaten die Erwartungen in neueren Studien nicht erfüllt und Heilungsraten von lediglich $31 \%$ (Plug [19]) bzw. $45 \%$ (OTSC [20]) gezeigt.

Die Fistulotomie wird gemäss den Leitlinien der European Crohn's and Colitis Organisation (ECCO) [21] als Therapie empfohlen, jedoch ist hier die Indikation zur Vermeidung einer Inkontinenz streng zu stellen.

Betreffend der Fistulotomie mit primärer Sphinkterrekonstruktion liegen keine isolierten Daten für Patienten mit einem Morbus Crohn vor.

Unabhängig von diesen Resultaten besteht ein nicht zu vernachlässigendes Risiko, dass bei Patienten mit einem zum Operationszeitpunkt floriden Morbus Crohn postoperativ schlecht heilende Wunden resultieren. Aus diesem Grund werden risikoreiche perianale Eingriffe bei diesem Patientengut von erfahrenen Chirurgen nur zurückhaltend durchgeführt.

\section{Funktionsmechanismus mesenchymaler Stammzellen}

Aus den oben genannten Gründen bedarf es neuer Behandlungsoptionen für Crohn-assoziierte Fisteln bei Patienten mit Morbus Crohn.

Die Verwendung mesenchymaler Stammzellen stellt einen neuen Therapieansatz dar, in den aufgrund der unbefriedigenden Alternativen grosse Erwartungen gesetzt werden.

Stammzellen können aus verschiedenen Geweben, wie dem Knochenmark, dem Fettgewebe, der Muskulatur oder der Nabelschnur, extrahiert werden. Sie sind pluripotent, sprich: Sie haben die Fähigkeit, sich in verschiedene Gewebe zu differenzieren. Dies hängt zum einem vom Stimulus und zum anderen von den Umgebungskonditionen ab, unter denen sie expandiert werden. Ihnen wird eine potenzielle immunmodulatorische Rolle zugesprochen und sie verfügen über wachstumsfördernde Fähigkeiten.

Im In-vitro-Modell verhindern die Stammzellen die Differenzierung von Monozyten in dendritische Zellen, daneben unterdrücken sie die Aktivierung und Proliferation der T-Lymphozyten [22]. Einige Studien zeigten darüber hinaus einen Anstieg der T-Zellen [23]. Der exakte Wirkungsmechanismus bleibt jedoch unklar.

Zusammenfassend kommt es durch die Kumulation der oben genannten Eigenschaften zu einer Reduktion der Entzündung und einer Heilung des Gewebes.

Die Extraktion der mesenchymalen Zellen aus dem Fettgewebe eignet sich am besten für die therapeutische Anwendung. Dies liegt zum einen an der einfachen Erreichbarkeit und zum anderen an der Option, eine möglichst grosse Anzahl an Zellen zu erhalten [24].

\section{Studienlage}

Insgesamt gibt es nur wenige prospektive Studien zur Behandlung Crohn-assoziierter Fisteln bei Patienten mit einem Morbus Crohn.

Eine der ersten Arbeiten zu Anwendung von Stammzellen wurde 2003 durch Garcia-Olmo et al. als Case Report beschrieben mit erfolgreichem Verschluss einer rektovaginalen Fistel [25]. In einer Folgestudie, einer Phase-I-Studie im Rahmen einer Fallserie mit einer verhältnismässig kleinen Fallzahl von 5 Patienten, konnte ein Verschluss der Fisteln bei $75 \%$ der Patienten gezeigt werden [26].

In der Folge wurden mehrere PhaseI- [27-29] und Phase-II-Studien [30, 31] durchgeführt. Darunter sind verschiedene Studien mit zum Teil nur kleinen Fallbeschreibungen und Arbeiten mit jeweils kleinen Fallzahlen von 1-43 Patienten $[28,32]$. Daneben entstanden einige wenige randomisierte Studien, vereinzelte Arbeiten weisen hier Patientenzahlen von über 200 eingeschlossenen Patienten auf $[30,33]$. In den RCT wird die Applikation der mesenchymalen Stammzellen entweder mit einem Placebo oder der Injektion von Fibrinkleber verglichen [30, 34].

Die durchgeführten Studien unterscheiden sich zum einen in dem Ursprung der verwendeten Stammzellen, die entweder aus dem Fettgewebe [25-27] oder aus dem Knochenmark [23, 29] gewonnen werden. Ebenfalls unterschieden wird in den Studien zwischen applizierten autologen $[28,34]$ oder allogenen $[29$, 30] Zellen und der verwendeten Menge der injizierten Zellen. Bislang gibt es jedoch keine Studie, die die Anwendung allogener bzw. autologer Stammzellen vergleicht.

In einigen Studien werden anstelle der Injektion von Einzeldosen wiederholte Applikationen beschrieben.

Das Nachkontrollintervall liegt in den Arbeiten ebenfalls in einer weiten Bandbreite von einem bis 72 bzw. 104 Monaten $[32,35]$.

Die meisten Studien betreffen die Behandlung Crohn-assoziierter perianaler Fisteln. Einige wenige Daten existieren zur Anwendung mesenchymaler Stammzellen bei enterokutanen oder rektovaginalen Fisteln und bei kryptoglandulären Fisteln [23, 33, 36].

In einem systematischen Review mit Metaanalyse von Ciccocioppo et al. wurden insgesamt 23 Publikationen (17 Studien und 6 Abstracts) analysiert. Kumulativ konnten so die Daten von 494 Patienten, die mit mesenchymalen Stammzellen behandelt wurden - davon 412 innerhalb von RCT [30, 33], mit $202 \mathrm{~Pa}$ tienten in den Vergleichsgruppen verglichen werden. Hierbei wurde aufgezeigt, dass bei den Crohn-assoziierten Fisteln in Rahmen der Beobachtungsstudien ein Fistelverschluss in bis zu $80 \%$ der Patienten erreicht werden konnte und im Rahmen der RCT in bis zu $64 \%$.

Der Fistelverschluss wurde in der Regel über eine Periode von 6 Monaten gemessen. Betrachtet man isoliert die RCT, zeigte sich in diesen eine $50 \%$ höhere Verschlussrate im Vergleich zum Kontrollarm ohne Stammzellinjektion [37].

Das Risiko der Applikation und die Rate an Nebenwirkungen ist insgesamt überschaubar. Die häufigsten Komplikationen waren perianale Abszesse (12\%) und Proktalgie (13\%) [30]. 
Schweiz. Gastroenterol. 2020 · 1:130-137 https://doi.org/10.1007/s43472-020-00026-8

(c) Der/die Autor(en) 2020

\section{Cabalzar-Wondberg $\cdot$ M. Turina}

\section{Mesenchymale Stammzellen als neue Therapie bei Morbus-Crohn-Patienten mit Fisteln}

\section{Zusammenfassung}

Die Behandlung komplexer Morbus-Crohn-assoziierter perianaler Fisteln erfordert meist ein interdisziplinäres Vorgehen. Der medikamentösen Therapie wird zur initialen Behandlung sowie zum Remissionserhalt eine essenzielle Rolle zuteil. Bei einem unzureichenden Ansprechen oder bei Therapieversagen ist bei symptomatischen Fisteln ein chirurgisches Vorgehen der nächste Schritt. Aufgrund der Komplexität der Grunderkrankung und der krankheitsassoziierten Anatomie führt die operative Behandlung jedoch häufig zu Fistelrezidiven. Die Behandlung mit mesenchymalen Stammzellen etabliert sich als eine neue innovative Therapieoption bei Patienten mit perianalen Crohn-assoziierten komplexen Fisteln, die auf eine konventionelle Therapie oder auf Biologika nicht oder nur unzureichend angesprochen haben. Die aus dem Fettgewebe eines Spenders gewonnenen Stammzellen werden lokal injiziert und weisen immunmodulatorische und antiinflammatorische Eigenschaften auf, was zu einer besseren Fistelheilung führen soll. Diese ambulant durchführbare Operation stellt zudem eine sphinkterschonende Behandlungsmethode ohne größere Wundfläche dar. In der Literatur finden sich zum jetzigen Zeitpunkt vielversprechende Resultate, die bislang auch im eigenen Patientengut bestätigt werden können.

Schlüsselwörter

Perianalfistel · Entzündliche Darmerkrankung · Allogeneic adipose-derived Stammzellen . Stammzelltherapie · Fistelverschluss

\section{Cellules souches mésenchymateuses en tant que nouveau traitement de la maladie de Crohn fistulante}

\section{Résumé}

Le traitement de fistules périanales complexes associées à la maladie de Crohn exige en général une approche interdisciplinaire. Le traitement médicamenteux devient le traitement initial et en partie joue un rôle essentiel pour maintenir la rémission. Lors de réponse insuffisante ou d'échec du traitement de fistules causant des symptômes, la prochaine étape est une intervention chirurgicale. En raison de la complexité de la maladie de base et de l'anatomie impliquée, le traitement chirurgical entraîne souvent toutefois le développement de fistules récidivantes.
Le traitement par des cellules souches mésenchymateuses s'établit en tant qu'option thérapeutique novatrice lors de non-réponse ou de réponse insuffisante au traitement conventionnel ou aux biomédicaments chez les patients souffrant de fistules périanales complexes dans le cadre de la maladie de Crohn. Les cellules souches prélevées dans le tissu adipeux d'un donneur sont injectées localement dans le site où leurs propriétés immunomodulatrices et anti-inflammatoires doivent permettre une meilleure guérison des fistules. Cette opération ambulatoire est en outre une méthode de traitement qui ménage le sphincter et ne cause pas une grande surface de plaie. La littérature décrit actuellement des résultats prometteurs que nous pouvons confirmer aussi chez notre propre patientèle jusqu'à présent.

Mots clés

Fistule périanale $\cdot$ Maladie inflammatoire de l'intestin · Cellules souches allogéniques dérivées de l'adiposité · Traitement aux cellules souches . Fermeture de fistules

\section{Cellule staminali mesenchimali come nuova terapia nei pazienti affetti da morbo di Crohn con fistole}

\section{Riassunto}

Di solito il trattamento delle fistole perianali complesse associate alla malattia di Crohn richiede un approccio interdisciplinare. La terapia farmacologica svolge un ruolo essenziale nel trattamento iniziale e nel mantenimento della remissione. In caso di risposta inadeguata o di fallimento della terapia, il passo successivo è l'intervento chirurgico per il trattamento delle fistole sintomatiche. Tuttavia, a causa della complessità della malattia sottostante e dell'anatomia associata a questa patologia, il trattamento chirurgico porta spesso alla recidiva della fistola. Il trattamento con cellule staminali mesenchimali si sta affermando come nuova opzione terapeutica innovativa per i pazienti con fistole perianali complesse associate al morbo di Crohn che non hanno risposto, o che hanno risposto in modo inadeguato, alla terapia convenzionale o ai biofarmaci. Le cellule staminali ottenute dal tessuto adiposo di un donatore vengono iniettate localmente e presentano proprietà immunomodulanti $\mathrm{e}$ antinfiammatorie, che dovrebbero portare ad una migliore guarigione della fistola. Inoltre, questo intervento, che può essere eseguito in regime ambulatoriale, rappresenta un metodo di trattamento delicato per lo sfintere e limita l'ampiezza della ferita. La letteratura attualmente contiene risultati promettenti, che possono essere confermati dai nostri pazienti.

\section{Parole chiave}

Fistola perianale $\cdot$ Malattia infiammatoria intestinale $\cdot$ Cellule staminali allogeniche derivate da adiposità · Terapia con cellule staminali · Chiusura della fistola
Betreffend die applizierte Zellzahl scheint eine mittlere Applikation von einmal $10^{7}$ bzw. 3-mal $10^{7}$ Zellen den höchsten Behandlungsnutzen $\mathrm{zu}$ zeigen, auch wiederholte Anwendungen mit guten Resultaten sind durchführbar [29].

Die aktuellsten Daten einer grossen Pilotstudie zur Verwendung von mesenchymalen Stammzellen in der Behandlung von Fisteln bei Patienten mit Morbus
Crohn stammen aus dem sog. ADMIRE Trial. Dies ist eine doppelblind randomisierte placebokontrollierte Multizenterstudie, die an 49 Kliniken europaweit und in Israel durchgeführt wurde. Eingeschlossen wurden volljährige Patien- 
Hier steht eine Anzeige.

\section{曾 Springer}




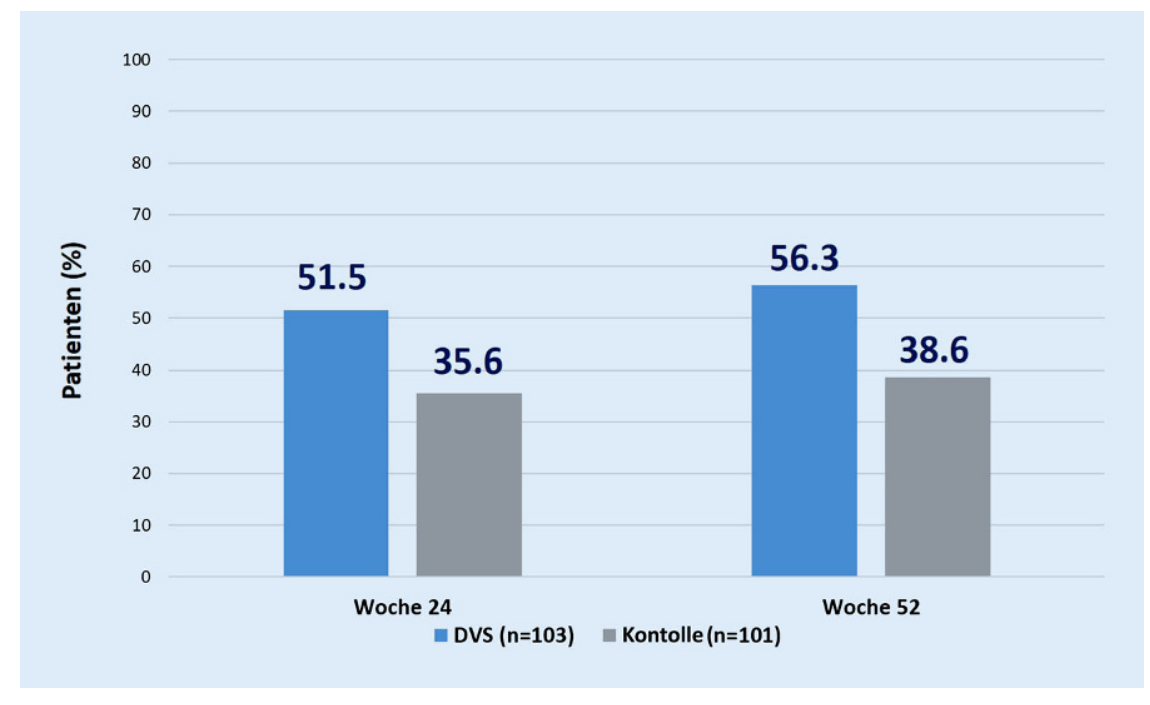

Abb. 1 A Resultate des ADMIRETrial: kombinierte Remission unter Stammzellen vs. Placebo in Woche 24 und 52 [38]. ADMIRE (Adipose Derived Mesenchymal Stem Cells for Induction of Remission in Perianal Fistulizing Crohn's Disease)

ten mit einem Morbus Crohn und komplexen perianalen Fisteln, die therapierefraktär auf mindestens eine konventionelle Therapie oder auf ein Biologikum waren. Insgesamt wurden $212 \mathrm{~Pa}$ tienten randomisiert, davon wurden 107 der Stammzellgruppe und 105 dem Kontrollarm zugeteilt. Bei der Intention-totreat (ITT)-Gruppe wurde ein chirurgischer Fistelverschluss mit Verschluss der inneren Fistelöffnung durchgeführt gefolgt von der einmaligen lokalen Injektion von 120 Mio. Stammzellen. In der Kontrollgruppe folgte dem chirurgischen Verschluss die Injektion eines Placebos.

Der primäre Endpunkt wurde mit der „combined remission“ sehr eng gewählt. Diese kombinierte Remission ist definiert als Kombination aus der klinischen Remission (Verschluss der behandelten Fistel und keine Sezernierung auf Kompression) und keiner nachweisbaren Flüssigkeitskollektion grösser als $2 \mathrm{~cm}$ im durchgeführten MRT.

In der postoperativen Woche $24 \mathrm{konn}$ te eine kombinierte Remission in der mit Stammzellen behandelten Patientengruppe von $51 \%$ vs. $36 \%$ gezeigt werden.

Diese Ergebnisse konnten in der Folgestudie bestätigt werden und zeigten auch in Woche 52 signifikante Werte mit einer kombinierten Remission von $56,3 \%$ in der Stammzell- vs. $38,6 \%$ in der Kontrollgruppe (• Abb. 1).
Bei den Patienten, die eine kombinierte Remission in Woche 24 erlangten, zeigten sich in Woche 52 weniger Rezidive als bei den Patienten in der Kontrollgruppe (75\% vs. $55,9 \%$ ).

Die klinische Remission wurde signifikant häufiger in der Gruppe der mittels Stammzellen behandelten Patienten erreicht $(59,2 \%$ vs. $41,6 \%)$

Unerwünschten Ereignisse traten bei $17 \%$ vs. $29 \%$ in der Kontrollgruppe auf. Auf häufigsten waren dies der Perianalabszess (6 in der Stammzellgruppe vs. 9 in der Kontrollgruppe) und die Proktalgie (5 vs. 9 Patienten; [30, 38]).

Es konnte auch nach 104 Wochen eine gleichbleibende klinische Remission von $56 \%$ vs. $40 \%$ dokumentiert werden [39].

Darüber hinaus konnte eine Verbesserung des Perianal Crohn's Disease Activity Index (PCDAI) gezeigt werden mit einem Anstieg des Gesamtscores in Woche 104 um 2,6 Punkte vs. 1,3 Punkte in der Kontrollgruppe. Die Autoren dieser Studie schlussfolgerten, dass die Behandlung mit mesenchymalen Stammzellen eine effektive Therapie komplexer perianaler Fisteln bei Morbus Crohn darstellt. Sie räumen jedoch auch ein, dass die Resultate aufgrund der limitierten Patientenzahl mit Vorsicht zu interpretieren sind.

\section{Behandlung mit mesenchy- malen Stammzellen in der Schweiz}

Das Medikament Darvadstrocel wurde durch Swissmedic im Dezember 2018 in der Schweiz zugelassen. Die Therapie mit adipösen mesenchymalen Stammzellen wurde im Februar 2019 schweizweit erstmalig am Universitätsspital Zürich durchgeführt und wird gegenwärtig an 2 Zentren in der Schweiz angeboten, dem Universitätsspital Zürich und am Centre hospitalier universitaire vaudois (CHUV).

Bei der Patientenselektion werden $\mathrm{Pa}$ tienten eingeschlossen, die unzureichend auf eine konventionelle Therapie oder auf Biologika angesprochen haben.

Patienten, die für eine Therapie mit mesenchymalen Stammzellen qualifizieren, sollten volljährig sein und an komplexen perianalen Fisteln aufgrund eines Morbus Crohn leiden. Es sollte keine oder lediglich eine moderate luminale Krankheitsaktivität vorliegen. Die Anzahl der Fistelöffnungen ist auf 2 interne und 3 externe Öffnungen beschränkt (• Abb. 2).

Präoperativ wird bei allen Patienten eine Magnetresonanztomographie des Beckens durchgeführt mit der Frage nach nicht drainierten Fistelgängen oder vorhandenen Abszessen. Sollten sich diese im MRT bestätigen, werden die Fistelgänge mittels Seton-Drainage für mindestens 6 Wochen konditioniert und Abszesse, die eine Grösse von $2 \mathrm{~cm}$ überschreiten, eröffnet.

Die Herstellung der Stammzellen der Firma Takeda Pharma AG (Glattpark [Opfikon]) unter dem Namen Alofisel $^{\circledR}$ beansprucht 16 Arbeitstage. Ist der Produktionsvorgang einmal gestartet, kann dieser nicht mehr unterbrochen werden und folglich das Operationsdatum nach Bestellung des Medikaments nicht mehr geändert werden. Aus diesem Grund werden in Zürich die Operationen idealerweise mit einem „Alternativpatienten“ geplant, der bereitsteht für den Fall, dass der ursprünglich vorgesehene Patient seine Operation unerwartet nicht wahrnehmen kann. Neben diesen logistischen und organisatorischen Herausforderungen ist der administrative Aufwand im Zusammenhang mit der Erlangung von 


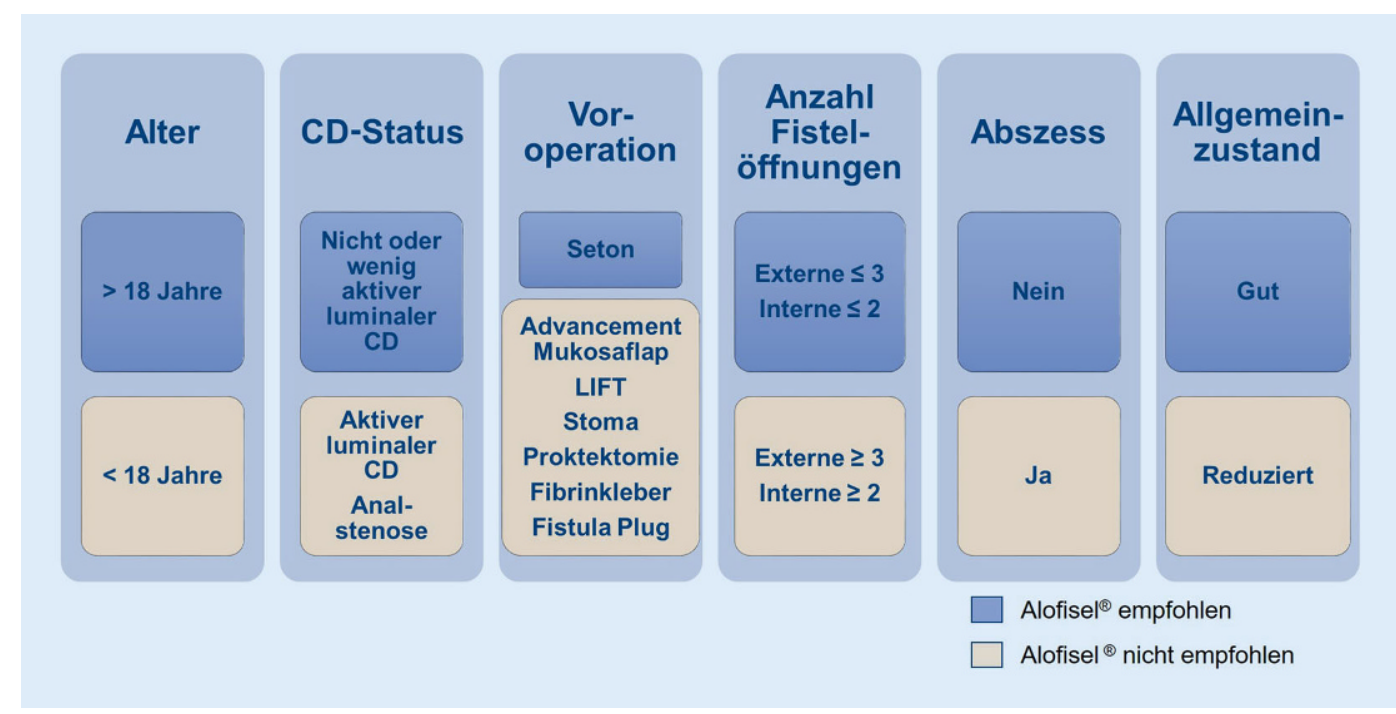

Abb. $2<$ Einschlusskriterien für die Behandlung mit mesenchymalen Stammzellen

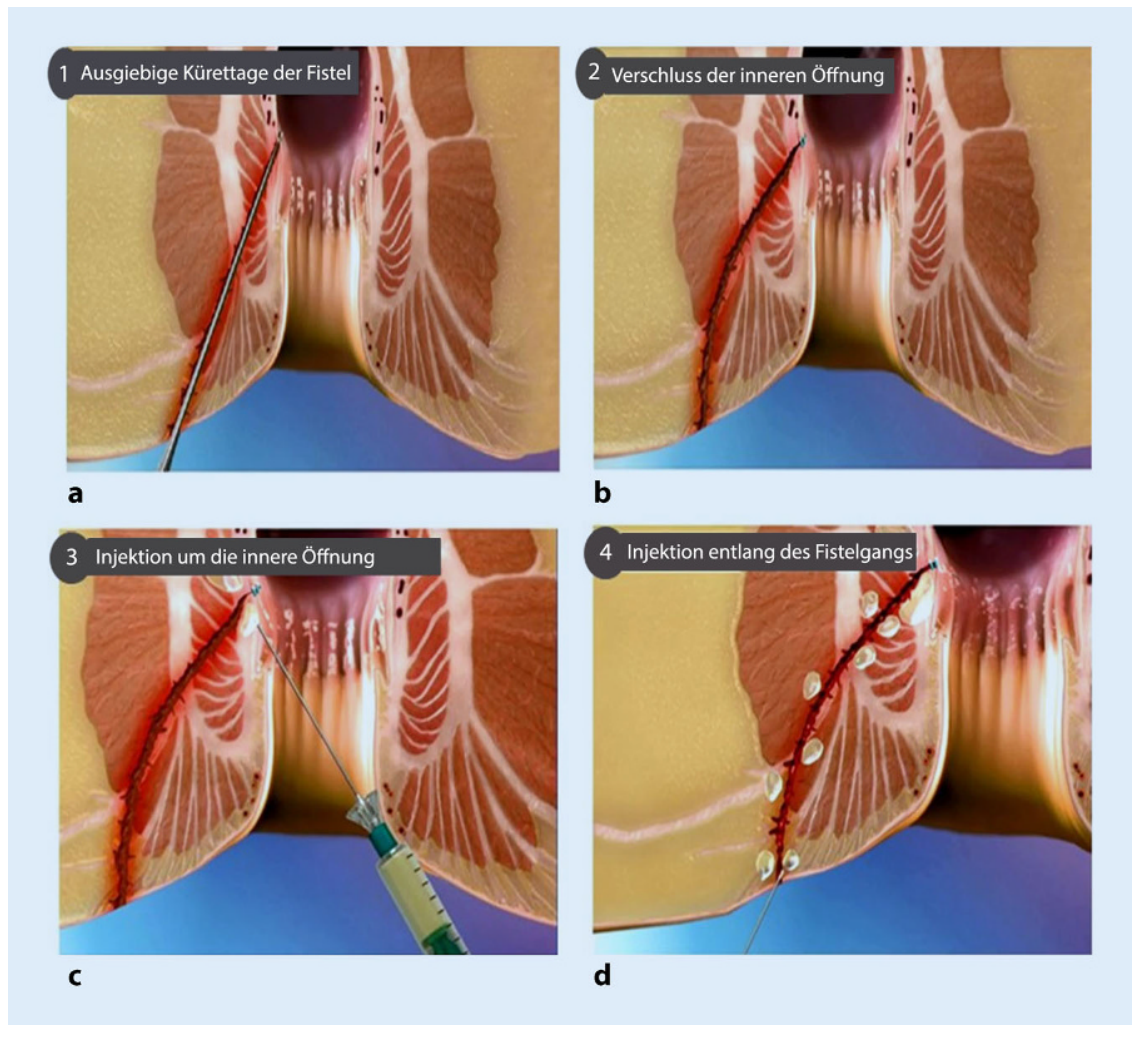

Abb. 3 ॥ Intraoperative Applikation. (Mit freundlicher Genehmigung von Takeda Pharma AG, Glattpark [Opfikon])

Kostengutsprachen nicht zu unterschätzen. Dies liegt einmal daran, dass das Medikament aktuell noch nicht auf der Spezialitätenliste geführt wird und der Preis bei ca. 70.000 CHF pro Anwendung liegt.

Nach der Extraktion der Zellen aus dem Fettgewebe des Spenders werden diese im Labor kultiviert.
Transporttemperatur konstant zwischen $15^{\circ}$ und $25^{\circ} \mathrm{C}$ lag.

Im Operationssaal führen wir routinemässig eine endoanalen 3-D-Sonographie durch zur aktuellen Standortbestimmung mit der Frage nach Seitengängen oder Kollektionen.

$\mathrm{Zu}$ Beginn der Operation wird die Seton-Drainage entfernt. Danach erfolgt eine ausgiebige Kürettage des Fistelgangs und der inneren Fistelöffnung. Der Fistelgang wird mit $\mathrm{NaCl}$ gespült und anschliessend wird die innere Fistelöffnung mit einer Vollwandnaht mit einem resorbierbaren Faden verschlossen. Der suffiziente Verschluss wird durch die erneute Injektion von $\mathrm{NaCl}$ über die äussere Fistelöffnung verifiziert.

Erst jetzt werden die Stammzellen aus der Transportbox entnommen und resuspendiert. Das Medikament wird in insgesamt 4 Glasfläschchen geliefert, die je 30 Mio. Zellen beinhalten. Dies entspricht einer kumulativen Dosis von 120 Mio. applizierten Zellen. Die Hälfte der Dosis wird submukös um die innere Fistelöffnung injiziert, die andere Hälfte in kleinen Depots in das direkt an den Fisteleingang angrenzende Gewebe. Die externe Fistelöffnung bleibt offen. Eine leichte Massage der externen Fistelöffnung beendet den Eingriff (• Abb. 3a-d).

Der Eingriff wird als ambulante Operation durchgeführt, sodass die Patienten das Krankenhaus 1-2 Stunden postoperativ verlassen können. 


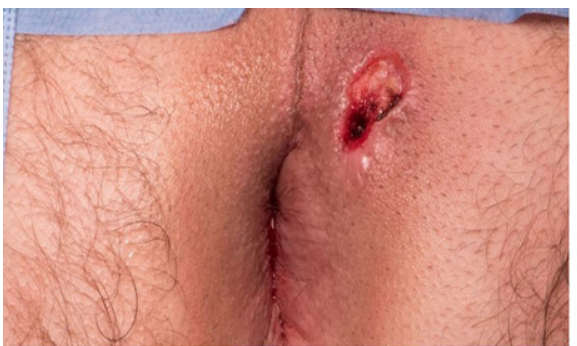

Postoperativ

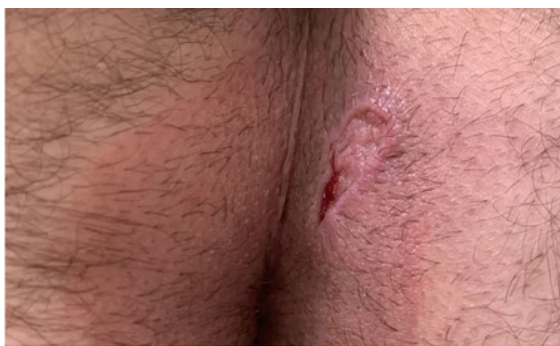

1 Woche p.o.

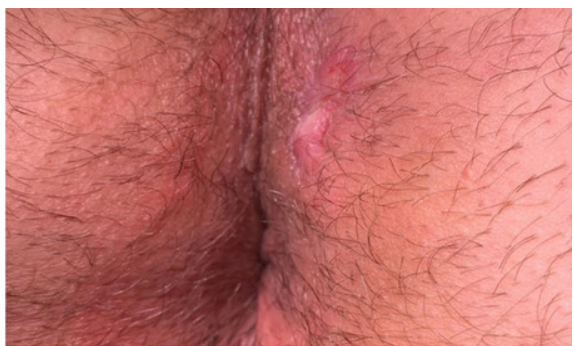

4 Wochen p.o.

Abb. 4 A Postoperativer Heilungsverlauf nach 1 und 4 Wochen

In der postoperativen Phase sollte von analer Manipulation abgesehen werden, eine gute Stuhlregulation ist zu beachten und die externe Wunde sollte abgeduscht werden. Ein Ausduschen in den Fistelgang hinein sollte jedoch vermieden werden.

Die postoperativen Nachkontrollen führen wir sehr engmaschig durch und sehen die Patienten nach 1, 2, 4 und 6 Wochen. Sollte sich die Wunde 6 Wochen postoperativ verschlossen haben, erfolgen weitere Kontrollen nach 3, 6 und 12 Monaten (• Abb. 4).

Die ersten klinikinternen Erfahrungen und Resultate sind vielversprechend mit einem schnellen Sistieren der Sekretion. Es fällt postoperativ eine rasche Wundheilung auf und die Patienten beschreiben, wenn überhaupt, nur diskrete postoperative Schmerzen.

Bei den mittlerweile 11 behandelten Patienten finden sich bei 8 Patienten komplett verheilte Fisteln. Zwei weitere Patienten befinden sich in klinischer Remission mit noch geringfügig oberflächlich offener Wunde. Bei bislang einem Patienten ist es zu einem Rezidiv gekommen.

\section{Fazit für die Praxis}

Die Behandlung komplexer perianaler Fisteln stellt nach wie vor eine Herausforderung dar. Ein interdisziplinärer Therapieansatz ist der Eckpfeiler einer erfolgreichen Behandlung. Bei Versagen oder unzureichendem Ansprechen auf die konventionelle Therapie oder auf Biologika kann bei symptomatischen perianalen Fisteln die Applikati- on der mesenchymalen Stammzellen evaluiert werden. Diese ist indiziert bei volljährigen Patienten mit einem nichtaktiven oder nur wenig aktiven luminalen Morbus Crohn. Die mesenchymalen Stammzellen fördern aufgrund ihrer immunmodulatorischen und antiinflammatorischen Eigenschaften die Abheilung der Fisteln in chronisch-entzündlich verändertem Gewebe, wie dies beim Morbus Crohn typisch ist. Weitere Studien zur klinischen Wirksamkeit der Stammzellen in der Behandlung dieser Fisteln sind jedoch notwendig bevor abschliessende Empfehlungen zur Patientenselektion respektive Indikationsstellung gemacht werden können.

\section{Korrespondenzadresse}

Dr. med. Daniela Cabalzar-
Wondberg
Klinik für Viszeral- und
Transplantationschirurgie,
Universitätsspital Zürich
Rämistrasse 100, 8006 Zürich,
Schweiz
daniela.cabalzar-
wondberg@usz.ch

Link für Überweisung von Patienten: http://www.vis. usz.ch/fachwissen/proktologie/seiten/fisteln.aspx

Funding. Open access funding provided by University of Zurich.

\section{Einhaltung ethischer Richtlinien}

Interessenkonflikt. D. Cabalzar-Wondberg und M. Turina geben an, dass folgender Interessenskonflikt besteht: Honorar von Takeda Pharma AG (Glattpark [Opfikon]) für Vortragstätigkeit.
Für diesen Beitrag wurden von den Autoren keine Studien an Menschen oder Tieren durchgeführt. Für die aufgeführten Studien gelten die jeweils dort angegebenen ethischen Richtlinien

Open Access. Dieser Artikel wird unter der Creative Commons Namensnennung 4.0 International Lizenz veröffentlicht, welche die Nutzung, Vervielfältigung Bearbeitung, Verbreitung und Wiedergabe in jeglichem Medium und Format erlaubt, sofern Sie den/die ursprünglichen Autor(en) und die Quelle ordnungsgemäß nennen, einen Link zur Creative Commons Lizenz beifügen und angeben, ob Änderungen vorgenommen wurden.

Die in diesem Artikel enthaltenen Bilder und sonstiges Drittmaterial unterliegen ebenfalls der genannten Creative Commons Lizenz, sofern sich aus der Abbildungslegende nichts anderes ergibt. Sofern das betreffende Material nicht unter der genannten Creative Commons Lizenz steht und die betreffende Handlung nicht nach gesetzlichen Vorschriften erlaubt ist, ist für die oben aufgeführten Weiterverwendungen des Materials die Einwilligung des jeweiligen Rechteinhabers einzuholen.

Weitere Details zur Lizenz entnehmen Sie bitte de Lizenzinformation auf http://creativecommons.org/ licenses/by/4.0/deed.de.

\section{Literatur}

1. Schwartz DA et al (2002) The natural history of fistulizing Crohn's disease in Olmsted County, Minnesota. Gastroenterology 122(4):875-880

2. Hellers $\mathrm{G}$ et al (1980) Occurrence and outcome after primary treatment of anal fistulae in Crohn's disease. Gut 21(6):525-527

3. Singh B et al (2004) Perianal Crohn's disease. Br Surg 91(7):801-814

4. Bell SJ et al (2003) The clinical course of fistulating Crohn's disease. Aliment Pharmacol Ther 17(9):1145-1151

5. Bergstrand O et al (1980) Outcome following treatment of anal fistulae in Crohn's disease. Acta Chir Scand Suppl 500:43-44

6. Mahadev $S$ et al (2011) Quality of life in perianal Crohn's disease: what do patients conside important? Dis Colon Rectum 54(5):579-585

7. Scharl M, Rogler G (2014) Pathophysiology of fistula formation in Crohn's disease. World J Gastrointest Pathophysiol 5(3):205-212 
8. Eglinton TW et al (2012) The spectrum of perianal Crohn's disease in a population-based cohort. Dis Colon Rectum 55(7):773-777

9. Parks AG, Gordon PH, Hardcastle JD (1976) A classification of fistula-in-ano. BrJSurg 63(1):1-12

10. Sandborn WJ et al (2003) AGA technical review on perianal Crohn's disease. Gastroenterology 125(5):1508-1530

11. Bouguen $\mathrm{G}$ et al (2013) Long-term outcome of perianal fistulizing Crohn's disease treated with infliximab. Clin Gastroenterol Hepatol 11(8):975-981e1-4

12. Molendijk I et al (2014) Disappointing durable remission rates in complex Crohn's disease fistula. Inflamm Bowel Dis 20(11):2022-2028

13. Dewint $P$ et al (2014) Adalimumab combined with ciprofloxacin is superior to adalimumab monotherapy in perianal fistula closure in Crohn's disease: a randomised, double-blind, placebo controlled trial (ADAFI). Gut 63(2):292-299

14. Soltani A, Kaiser AM (2010) Endorectal advancement flap for cryptoglandular or Crohn's fistula-inano. Dis Colon Rectum 53(4):486-495

15. Rozalen Vetal (2017) Advancement flap technique for anal fistula in patients with Crohn's disease: a systematic review of the literature. Cir Esp 95(10):558-565

16. Stellingwerf ME et al (2019) Systematic review and meta-analysis of endorectal advancement flap and ligation of the intersphincteric fistula tract for cryptoglandular and Crohn's high perianal fistulas. BJSOpen 3(3):231-241

17. Gingold DS, Murrell ZA, Fleshner PR (2014) A prospective evaluation of the ligation of the intersphincteric tract procedure for complex anal fistula in patients with Crohn's disease. Ann Surg 260(6):1057-1061

18. van Praag EM et al (2020) Ligation of the intersphincteric fistula tract and endorectal advancement flap for high perianal fistulas in Crohn's disease: a retrospective cohort study. JCrohns Colitis 14(6):757-763

19. Senejoux $A$ et al (2016) Fistula plug in fistulising ano-perineal Crohn's disease: a randomised controlled trial.J Crohns Colitis 10(2):141-148

20. Prosst RL et al (2012) The anal fistula claw: the OTSC clip for anal fistula closure. Colorectal Dis 14(9):1112-1117

21. Adamina M et al (2020) ECCO guidelines on therapeutics in Crohn's disease: surgical treatment. JCrohns Colitis 14(2):155-168

22. Nauta AJ, Fibbe WE (2007) Immunomodulatory properties of mesenchymal stromal cells. Blood 110(10):3499-3506

23. Ciccocioppo R et al (2011) Autologous bone marrow-derived mesenchymal stromal cells in the treatment of fistulising Crohn's disease. Gut 60(6):788-798

24. Valencia J et al (2016) Comparative analysis of the immunomodulatory capacities of human bone marrow-and adipose tissue-derived mesenchymal stromal cells from the same donor. Cytotherapy 18(10):1297-1311

25. Garcia-Olmo D et al (2003) Autologous stem cell transplantation for treatment of rectovaginal fistula in perianal Crohn's disease: a new cell-based therapy. Int J Colorectal Dis 18(5):451-454

26. Garcia-Olmo D et al (2005) A phase I clinical trial of the treatment of Crohn's fistula by adipose mesenchymal stem cell transplantation. Dis Colon Rectum 48(7):1416-1423

27. Dietz $A B$ et al (2017) Autologous mesenchyma stem cells, applied in a bioabsorbable matrix, for treatment of perianal fistulas in patients with Crohn's disease. Gastroenterology 153(1):59-62

28. Lee WY et al (2013) Autologous adipose tissuederived stem cells treatment demonstrated favorable and sustainable therapeutic effect for Crohn's fistula. Stem Cells 31(11):2575-2581

29. Molendijk I et al (2015) Allogeneic bone marrowderived Mesenchymal Stromal cells promote healing of refractory perianal fistulas in patients with Crohn's disease. Gastroenterology 149(4):918-927e6

30. Panes J et al (2016) Expanded allogeneic adiposederived mesenchymal stem cells (Cx601) for complex perianal fistulas in Crohn's disease: a phase 3 randomised, double-blind controlled trial. Lancet 388(10051):1281-1290

31. Cho YB et al (2013) Autologous adipose tissuederived stem cells for the treatment of Crohn's fistula: a phase I clinical study. Cell Transplant 22(2):279-285

32. Garcia-Olmo D et al (2010) Adipose-derived stem cells in Crohn's rectovaginal fistula. Case Rep Med 2010:961758. https://doi.org/10.1155/2010/ 961758

33. Herreros MD et al (2012) Autologous expanded adipose-derived stem cells for the treatment of complex cryptoglandular perianal fistulas: a phase III randomized clinical trial (FATT 1: fistula Advanced Therapy Trial 1) and long-term evaluation. Dis Colon Rectum 55(7):762-772

34. Garcia-Olmo D et al (2009) Expanded adiposederived stem cells for the treatment of complex perianal fistula: a phase II clinical trial. Dis Colon Rectum 52(1):79-86

35. Ciccocioppo Ret al (2015) Long-term follow-up of Crohn disease fistulas after local injections of bone marrow-derived mesenchymal stem cells. Mayo Clin Proc 90(6):747-755

36. Garcia-Arranz M et al (2016) Treatment of Crohn's-related rectovaginal fistula with allogeneic expanded-adipose derived stem cells: a phase I-lla clinical trial. Stem Cells Transl Med 5(11):1441-1446

37. Ciccocioppo R et al (2019) Systematic review with meta-analysis: Safety and efficacy of local injections of mesenchymal stem cells in perianal fistulas. JGH Open 3(3):249-260

38. Panes J et al (2018) Long-term efficacy and safety of stem cell therapy (Cx601) for complex perianal fistulas in patients with Crohn's disease. Gastroenterology 154(5):1334-1342e4(e4)

39. Adamina M et al (2020) The 13th European colorectal congress (\#ECCstGallen), 1-5 december 2019, St Gallen, Switzerland: congress report. Tech Coloproctol 24(6):619-628

Hinweis des Verlags. Der Verlag bleibt in Hinblick auf geografische Zuordnungen und Gebietsbezeichnungen in veröffentlichten Karten und Institutsadressen neutral.
Gaisser, Andrea, Weg-Remers, Susanne Patientenzentrierte Information in der onkologischen Versorgung

Evidenz und mehr

Berlin/Heidelberg: Springer-Verlag GmbH 2020, 1. Auflage, (ISBN: 978-3662-60460-1), 44,99 EUR

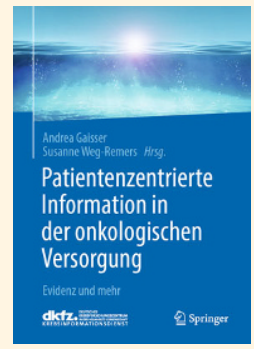

In Zusammenarbeit mit dem Krebsinformationsdienst ist im SpringerVerlag ein neues Buch erschienen. Es richtet sich an Ärztinnen

und Ärzte sowie Angehörige aller Gesundheitsberufe, die an der Versorgung von Krebspatienten beteiligt sind.

Das Buch „Patientenzentrierte Information in der onkologischen Versorgung" fasst die über Jahrzehnte gesammelten Erfahrungen des Krebsinformationsdienstes des Deutschen Krebsforschungszentrums im Umgang mit individuellen Anfragen von Krebspatienten zusammen und bietet Unterstützung beim Beantworten typischer und manchmal auch schwieriger Fragen zu den für Patienten wichtigen Themen.

Die Buchbeiträge folgen den Fragen, die sich viele Patientinnen und Patienten auf ihrem Weg durch die Krebserkrankung stellen. Illustriert anhand beispielhafter Anfragen werden ihre Bedeutung und praktische Relevanz für Patienten ebenso dargestellt wie die jeweilige gesicherte Datenlage. Zu jedem Kapitel sind weiterführende Informationen, auch für Patienten, und eine Literaturauswahl zusammengestellt. Eine Einführung zu den Herausforderungen der Informationsvermittlung und Kommunikation in der Onkologie sowie eine Auswahl nützlicher Hinweise und Empfehlungen für Informationssuche, Informationsbewertung und Kommunikation runden den Inhalt des Buches ab. 